\title{
Real-time Decision Support in Online Training Environments
}

\author{
Liz Bacon, $\mathrm{PhD}^{1}$, Lachlan MacKinnon, $\mathrm{PhD}^{1}$, and David Kananda, $\mathrm{BSc}^{1}$ \\ ${ }^{1}$ University of Greenwich, UK, e.bacon@gre.a.uk, L.Mackinnon@gre.ac.uk, D.M.Kananda@gre.ac.uk
}

\begin{abstract}
Multi-agency crisis management represents one of the most complex real-world situations, requiring rapid negotiation and decision-making under extreme pressure. However, the training provided to Gold Commanders (strategic planners), typically lacks the stress of a real crisis and research tells us that behaviour and decision-making are significantly affected by stress. It is therefore vital that training puts trainees under the pressure of a real crisis situation as far as is possible. The Pandora+ system has been developed to provide a realistic, immersive, augmented reality training environment in which the stress of each individual trainee can be managed by the trainer, during a training event, with the support of system intelligence. The system uses AI planning techniques to manage an unfolding crisis scenario, modelled as an event network which can be dynamically updated by the trainer during a training event. This modelling includes points of decision for trainees managed by automated rules from a knowledge base, behavioural modelling of the trainees, and ambient management of the environment to provide affective inputs to control and manage trainee stress. In this context, the system controls and reacts to trainee performance in relation to the events and decision points and can dynamically remodel and reconfigure the event network to respond appropriately to trainee decisions. The environment can also represent any missing trainees within the scenario and has the potential to provide training in any domain where an unfolding scenario of events are required for training.
\end{abstract}

Keywords-eLearning, Decision Support, Smart Environment, Crisis management training environment, timeline-based event network.

Digital Object Identifier (DOI): http://dx.doi.org/10.18687/LACCEI2015.1.1.192

ISBN: 13 978-0-9822896-8-6

ISSN: $2414-6668$

13 ${ }^{\text {th }}$ LACCEI Annual International Conference: “Engineering Education Facing the Grand Challenges, What Are We Doing?” July 29-31, 2015, Santo Domingo, Dominican Republic

DOI: http://dx.doi.org/10.18687/LACCEI2015.1.1.192 


\title{
Real-time Decision Support in Online Training Environments
}

\author{
Prof Liz Bacon, $\mathrm{PhD}^{1}$, Prof Lachlan MacKinnon ${ }^{1}, \mathrm{PhD}$, David Kananda, BSc (Hons) ${ }^{1}$ \\ University of Greenwich, UK, e.bacon@gre.a.uk, L.Mackinnon@gre.ac.uk, D.M.Kananda@gre.ac.uk
}

\begin{abstract}
Multi-agency crisis management represents one of the most complex real-world situations, requiring rapid negotiation and decision-making under extreme pressure. However, the training provided to Gold Commanders (strategic planners), typically lacks the stress of a real crisis and research tells us that behaviour and decision-making are significantly affected by stress. It is therefore vital that training puts trainees under the pressure of a real crisis situation as far as is possible. The Pandora ${ }^{+}$system has been developed to provide a realistic, immersive, augmented reality training environment in which the stress of each individual trainee can be managed by the trainer, during a training event, with the support of system intelligence. The system uses AI planning techniques to manage an unfolding crisis scenario, modelled as an event network which can be dynamically updated by the trainer during a training event. This modelling includes points of decision for trainees managed by automated rules from a knowledge base, behavioural modelling of the trainees, and ambient management of the environment to provide affective inputs to control and manage trainee stress. In this context, the system controls and reacts to trainee performance in relation to the events and decision points and can dynamically remodel and reconfigure the event network to respond appropriately to trainee decisions. The environment can also represent any missing trainees within the scenario and has the potential to provide training in any domain where an unfolding scenario of events are required for training.
\end{abstract}

Keywords-eLearning, Decision Support, Smart Environment, Crisis management training environment, timeline-based event network.

\section{INTRODUCTION}

The Pandora ${ }^{+}$Training Environment has been developed based on the output of an EU FP7 project called Pandora which ran between 2010 and 2012. It is a smart eLearning environment designed to train Strategic Level (Gold) Commanders, who may deal with crises at an international, national or local level, in crisis management Gold Commanders typically may have senior executive level or management responsibility for services involved in crisis management, such as police, fire and health authorities, or be local government executives with direct responsibility for protecting the functions of civil society. Crises in which Gold Commanders will be involved will typically present as complex situations, requiring a coordinated, multi-agency response, $9 / 11$ being one such example.
The role of a Gold Commander is explicitly strategic. They are in overall control of the emergency for their organization, however they will not generally be at the site of the emergency, but typically co-located with other Gold Commanders in a control room. They will propose solutions and set the direction for the tactical (Silver) Commanders to implement who will also typically not be physically present at the site of the emergency. Silver Commanders give direction to operational commanders (Bronze) who are responsible for organizing resources on the ground. In practice some of these roles may become blurred and Gold Commanders could also have some tactical or operational responsibility.

Whilst Gold Commanders will bring a wealth of experience and knowledge from their own specific areas, they typically have little understanding of how Gold Commanders in other agencies work and therefore need training to develop their skills in working with senior executives from other agencies to understand the constraints, culture, behavior and priorities etc. of those agencies. The importance of team training and the development of good communication skills cannot be over-emphasized and can be a matter of life and death [1]. A key component of this training includes the development of negotiation and communication skills [2] and the ability to understand the need for pragmatic trade-offs between agencies that may be necessary in order to manage and lead a crisis situation as a team. Desired outcomes from a training event, can include:

- Development of collaborative skills, including an understanding of how people work in teams and negotiate solutions to deliver decisions and solutions in a timely manner.

- Assessment of interpersonal skills, flexibility and ability to work with other Gold Commanders.

- Helping teams think about alternative solutions to solve problems, forcing trainees to move away from their preprepared plans and to think of innovative solutions to problems in a time-constrained and stressful environment

- Consideration of the impact of decisions on other services / agencies.

- Assessment of trainee performance in making decisions under pressure.

- Preparation of trainees to deal with the media, which is inevitable in the event of a crisis.

- Helping trainees to recognise their assumptions, challenge each other's, and to justify them as being reasonable at a specific point in time. These will be scrutinized after the

$1^{\text {th }}$ LACCEI Annual International Conference: “Engineering Education Facing the Grand Challenges, What Are We Doing?” 
event if a situation worsens and will inevitably be the focus of a post-crisis analysis.

- Monitoring the risk taking behaviour of the group, given research suggests that risk taking behaviour increases in a group situation [3].

- Reflecting on whether decisions made by the group made the situation worse or better.

In order to develop strategic plans to deal with crisis situations, as identified above, Gold Commanders are expected to work together to produce effective plans. However, pre-prepared strategic plans are typically developed in isolation from other agencies in the calm of an office environment. These plans will outline approaches, use of resources etc. and whilst such plans are obviously essential to preparing for a crisis, all crisis situations will present Gold Commanders with some unique circumstances and problems to solve, which could include stressful situations such as the requirement to make life and death decisions under extreme pressure and in a time constrained manner. Because different agencies will have different cultures and different priorities in the event of a crisis, which may clash, there is a need for multi-agency training to help people in different agencies understand each other and their priorities in advance and hopefully, through the use of realistic training scenarios, understand each better prior to a real crisis.

Gold Commanders are generally involved in a crisis when an event threatens human life, health, property, the critical national infrastructure or there is likely to be an impact on the supply of essential services. Their focus is to:

- Save and protect life whilst ensuring the health and safety of their own staff

- Relieve suffering

- Contain the emergency

- Provide information to the public

- Safeguard the environment

- Protect property

- Maintain/restore critical services

- Maintain normal services appropriately

- $\quad$ Promote and facilitate self-help

- Facilitate the investigation/inquiry

- Facilitate community recovery

- Evaluate and identify lessons learned.

Crisis management exercises and simulations must be a key component of preparing teams to respond in the event of a crisis, however they are typically done in the following ways.

a. Tabletop exercises. The cheapest and quickest approach to training is to design a crisis scenario, sit someone from each agency around a table, given them a written briefing and ask them to imagine the scenario described and then discuss in the team how they would solve it. These sessions are managed by a trainer who would try to emotionally engage the group with the description of the crisis that they have been presented with. In addition, the trainer may be required to playing the part of a missing agency person if that role was crucial to the exercise. They will also occasionally interject an event to represent an unfolding scenario after the initial event(s) which triggered the crisis, to see how the participants react and how their plans might change as the crisis scenario unfolds. The occasional media clip might be played to help make the exercise feel more real however, the nature of the exercise predominantly involves discussion by the trainees, around a table, as they try to solve the crisis. These exercises therefore lack the stress and pressure of the real event, and the impact and feedback on decisions taken in real time.

b. Real-world exercises. These are designed to simulate part of a particular crisis scenario that might occur. The benefit of these are that they are extremely realistic however, they are typically very expensive and time consuming to set up and run, and can only simulate a small part of a potential crisis.

c. Computer-based training environments. These simulate a crisis situation and provide a more realistic training environment than a tabletop exercise however, scenarios can be expensive to author and their ability to adapt during a training session can be limited.

In all of the above training situations, the approaches and products available provide a limited number of outcomes that can be simulated in all cases and this is one of the key requirements that the Pandora ${ }^{+}$training tool is designed to address. It does this by providing an immersive environment which delivers real-time, adaptable simulations with multiple outcomes that can be dynamically managed by a trainer, onthe-fly, during a training session, to allow a group of trainees to explore a train of thought and follow it through to the end, as opposed to being steered towards a limit number of fixed outcomes.

The remainder of this paper explores current approaches to crisis training and then describes the Pandora+ training environment, focusing on the real-time decision making support within the tool. It then briefly addresses evaluation before discussing conclusions and future work.

\section{LITERATURE REVIEW}

In recent years there has been a trend towards the use of computer based environments to provide a realistic, engaging. immersive and flexible training at a reasonable cost. The importance of realistic crisis management training cannot be over-emphasised, as in stressful situations, emotions can cause a wide range of effects on people's attention, perception and the cognitive processes involved in decision making, problemsolving and learning. Evidence suggests that individuals under stress and anxiety often fail to adopt rational-choice models. They often devote insufficient time to the 
consideration of available alternatives and / or consider alternatives in a disorganised manner, making decisions before considering all potential information [4]. It is therefore important to ensure that trainee crisis managers experience team working skills in stressful situations [5].

Computer training environments are typically based on games and / or simulation technologies for a number of reasons. Games as learning tools have been well recognised as beneficial for a long time [6] and the use of games in recent times, particularly for the training of healthcare professionals to manage crisis situations, has become commonplace [7], [8]. Games can also help to manage the cognitive load [9] which in turn helps people to manage their stress. However, the difference between games and simulations is not clear. From a survey undertaken by [10], some people view games as a subset of simulations, others take the opposite view, and some suggest that serious games (a game whose primary purpose is not entertainment) could be considered to be the intersection of the two. A variety of computer-based games and simulation environments have been developed for crisis management training. For example, commercial tools such as Hydra and Minerva [11] which are focussed around police and fire rescue simulations, and Vector Command [12] which has developed a command and control system and associated training. There are also a variety of training tools developed by research projects such as CRISIS [13].

All of these training environments offer a variety of features, tools and facilities and can provide effective crisis management training within pre-determined and fixed scenarios. However, none of them offer the combination or sophistication provided by the state-of-the-art Pandora $^{+}$ training environment [14], [15], the details of which are explained in the next section.

\section{THE PANDORA ${ }^{+}$TRAINING ENVIRONMENT}

The Pandora ${ }^{+}$environment is an augmented reality environment (real world is augmented with computergenerated input e.g. images, audio, video) as opposed to virtual reality (user is immersed in a virtual world which has replaced the real one). During the design process it was determined that this type of environment was more suited to the background and experience of a typical Gold Commander, and maintains a more realistic environment than a virtual world. However, this may change in the future as the current Gold Commander demographic changes and new personnel moving into those roles become more familiar with virtual worlds. The system has been designed to provide a learner centric, constructivist approach to education as opposed to teacher centric, instructivist approach.

As described above there are a number of different training tools in the market, however what makes Pandora+ unique is the combination of the following features, most of which are innovative in this space:

1. Scenario generation by a trainer - a scenario description, coupled with its multimedia assets, can be input either directly into Pandora ${ }^{+}$using an editor or uploaded through a spreadsheet and run at the push of a button. The trainer can configure Pandora ${ }^{+}$to the number of players and specify the roles of each player within the scenario. In other tools, generation of a scenario is not typically something a trainer can do, it is usually a bespoke and expensive activity.

2. Mode of delivery - can be used by a single trainee or as a multi-user training environment, in a fixed location where trainees are physically co-located, or distributed when trainees are dispersed.

3. Presentation of information - provides a variety of formats e.g. audio, video, texts, email, graphics and text. Group information can be presented through a multiscreen display if useful.

4. Use of Non-Player Characters (NPCs) - These are computer-based actors within the scenario that either provide an automated, fixed, pre-scripted representation of one of the roles outlined below, or can be taken over by the trainer to provide a dynamic capability within the scenario to follow through a train of thought by the trainees. The NPCs can be configured to demonstrate a variety of emotions and characteristics etc. and persist for the lifetime of the training, playing a full part in the training. Pandora ${ }^{+}$defines four types of NPC that can be rendered within the simulation environment.

i. HICON (Higher Control Strategic Agents) - these represent the most senior authority figures within the society e.g. Government Ministers who would be above the level of Gold Commanders, have the authority to demand actions or constrain resources, and could impose their decisions on the crisis team. They may also provide confidential information to one or more trainees who have to respond accordingly.

ii. LOCON (Lower Control Tactical Agents) - these represent the lower levels of command within the crisis team, and can provide valuable feedback on the tactical level realisation of the current strategy, as well as onsite reports of the physical situation, resources etc.

iii. External Experts - these represent specialists in particular areas of importance in a given scenario, and can be consulted by the trainees directly, or can indirectly provide inputs through media interviews or information sites.

iv. Missing trainees - for each scenario a set of key players are identified, and these must be represented in order that all the elements of the scenario can be realised. Each of these players is modelled as an NPC, with pre-determined actions in relation to the narrative of the scenario. If a trainee is missing, the relevant NPC(s) can be configured to take their place and enable 
the training event to take place. As a result a training event can run with any number of human trainees and will always have a full complement of players for the scenario.

5. Scenario management - the ability to pause the scenario during execution and rollback the scenario to a specific point in time in order allow trainees to try again, amend the scenario on the fly during training etc.

6. Full debrief post training - all communications (audio, video, texts, email, graphics and text etc.) and decisions are recorded and can be replayed for reflection and debriefing of trainees.

7. Stress management - This is the most significant feature of Pandora ${ }^{+}$which is different to other environments and is based around the intelligent support and management of trainee stress during a training exercise [14], [15]. The trainer may decide during a training session that one or more trainees are too relaxed or too stressed, and that the stress levels for one or more trainees should be increased or decreased, to better reflect a real world crisis scenario. As shown in Fig. 1, there are several features which can be used to assess and manipulate the stress of individual trainees or the group of trainees which use a mix of relevant features from both games and simulations.

\section{Stress Inputs:}

a. Behavioral modeling - human factors / psychological variables of trainees (e.g. Personality traits, leadership style, background experience, selfefficacy, stress \& anxiety), which have all been shown to have an influence on decision making under stress, are both modeled in advance of the training and monitored during training.

b. Biometric sensors, such as a heart rate monitor. Pandora $^{+}$can receive biometric information on each trainee which the trainer can monitor to judge stress levels, or can be used to provide automatic feedback to the system to provide input in order to ascertain if the target stress levels for individual trainees are correct.

c. Self-reporting by trainees during the training event

d. Trainer observation during the training event

\section{Stress Controls:}

a. Add and delete events as the scenario plays out

b. Alter decision points and option choices.

c. Increase or decrease the speed at which the scenario is played out relative to real time.

d. Adjust resources available for the trainees to use in solving their part of the crisis e.g. limit the number of fire trucks and crews to disperse.

e. Change deadlines to increase / decrease time pressures on trainees.

f. Set different challenges / goals for different trainees e.g. how they manage their resources, rules can be defined which have quantifiable outcomes, and levels of difficulty can also be set. These can also be updated during scenario execution.

g. Provide the Pandora ${ }^{+}$system with a target level of stress for one or more trainees which can be changed during scenario execution. Pandora ${ }^{+}$manages this through its affective state framework which can manipulate stress in two ways: through NPC behaviour or through the use of mashup rules to mix various multimedia assets in order to induce, or lower, stress e.g. overlay audio over a picture of a flooded hospital to either report the water is receding (to lower stress) or that the water is rising (to induce stress).

To summarize, the Pandora ${ }^{+}$system is a toolset providing a rich multimedia training environment, into which a scenario can be uploaded and executed. Both the trainer and trainee environments have been developed in Java so are platform neutral, and although designed for crisis management training, they can be used for any training situations that are eventbased i.e. a scenario in which trainees are required to respond to a series of unfolding events which occur over a period of time. A non-crisis management example might be training in trading strategies for buy stocks and shares on the stock market, as it responds to different world events that unfold over a period of time.

Stress management is a major feature of Pandora ${ }^{+}$ however this is predominantly associated with trainee decision making behaviour. Indeed, a key factor in the underpinning technology and algorithms used to provide the level of flexibility and dynamic behavior available in Pandora ${ }^{+}$is the manipulation of the event-network and its ability to manage multiple outcomes depending on the decisions that trainees make. This is a complex area and is a key focus of this paper. Details of this aspect of Pandora ${ }^{+}$are given in the next section

\section{DECISION MANAGEMENT}

The planning process generates an initial event network that aims to deliver, and is consistent with, the starting goals. Events are represented by a predicate identifying a time interval for the event, a start time and an end time. All events have a cause and relationships to other events which must be defined. The event network is essentially a hypergraph with the events representing nodes and the relations being the hyperedges [16].

Updated goals and new events, either interjected by the trainer directly or by the Emotion Engine (which places new events on the network in order to raise or lower stress), cause the AI planner to review the event network after each planning / execution cycle. A key role of the AI planning component is to check that these causal relations remain consistent after the 
new inputs have been included on the event network for execution, and as decisions by trainees steer the scenario through various branches of the decision tree towards a potentially infinite range of outcomes. Note that new events interjected by the trainer during execution may yield new branches of the decision tree which require the plans to be adapted on the fly and these updates to the event network must include consistency checks.

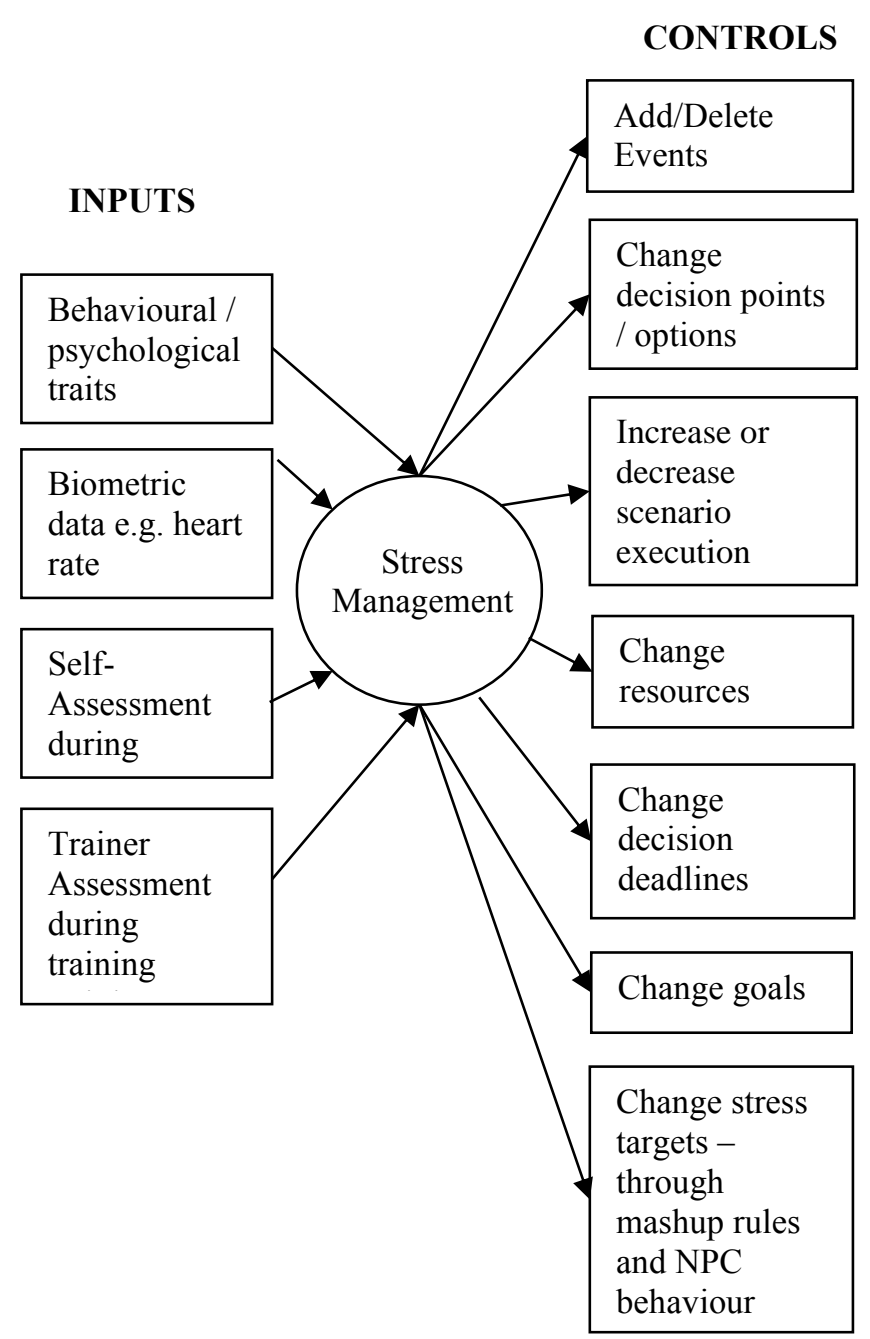

Fig. 1 Stress Management in Pandora ${ }^{+}$

If it appears that a rule cannot be applied as it would render an inconsistency, for example by violating a constraint, then the event network is returned to a previously consistent state and the planning proceeds from there. If a planning process is successful i.e. the event network is consistent with the goals provided and no constraints have been violated, then the plan is executed. Each communication channel is modelled on its own timeline as shown in Fig. 2.

\section{DECISION BRANCHING MECHANISM}

As described in the previous section, the scenario is modelled using a decision tree structure, when events require trainees to make decisions. This follows a standard decision tree model, where options provide branches on the event network, and to avoid proliferation of options complex decisions may be broken down into a number of steps. In crisis management, the relative value or cost of each option will be calculated by the trainees in terms of resource commitment relative to impact and outcomes, determined against the focus list described in section I.

Branching is the mechanism by which Pandoradynamically loads new events based on a selected decision event option during a scenario exercise. A Decision event is an event with a set of 2-4 options to choose from. Each option has consequences which lead to a further set of events. Essentially, the branching mechanism provides a means for Pandora ${ }^{+}$to simulate and deliver consequences based on a particular decision taken by the trainees. This mechanism is controlled by the branching model, which is as follows, also see Fig 3.

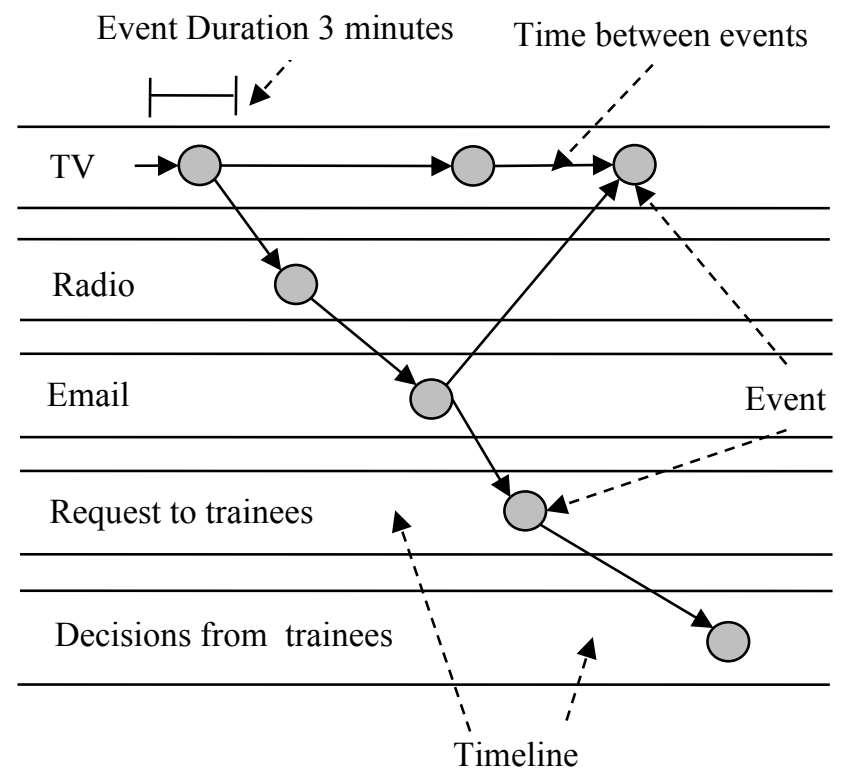

Fig. 2 Example of timelines and events

User view of the Branching Algorithm:

1) Decision event is dispatched to trainees

2) The trainee(s) choose their option

2.1) The trainer is notified of the response

2.2) Trainer agrees to proceed with exercise (agreement is not automatic in case the trainees opt to take their own path as opposed to a pre-determined one in which case the trainer will have to manage the unfolding scenario directly)

3) Get selected option response sent back in (2) 
4) Retrieve all events associated with selected option from the database (see Event Loading Algorithm below) up to next decision event.

5) Scenario execution is paused briefly whilst the next set of events are loaded. The play button will be unpressed ready to be pressed. Whilst retrieving events, there is a progress dialog which appears and disappears when all events are retrieved.

5.1) Display list of events in the scenario table below the previously executed events

Note that all relevant assets e.g. videos, documents etc. are downloaded to each trainee machine for local access at the beginning of the scenario (To avoid disruptions e.g. a slow download of a video file while the exercise is running). The decision branching mechanism does not download any assets.

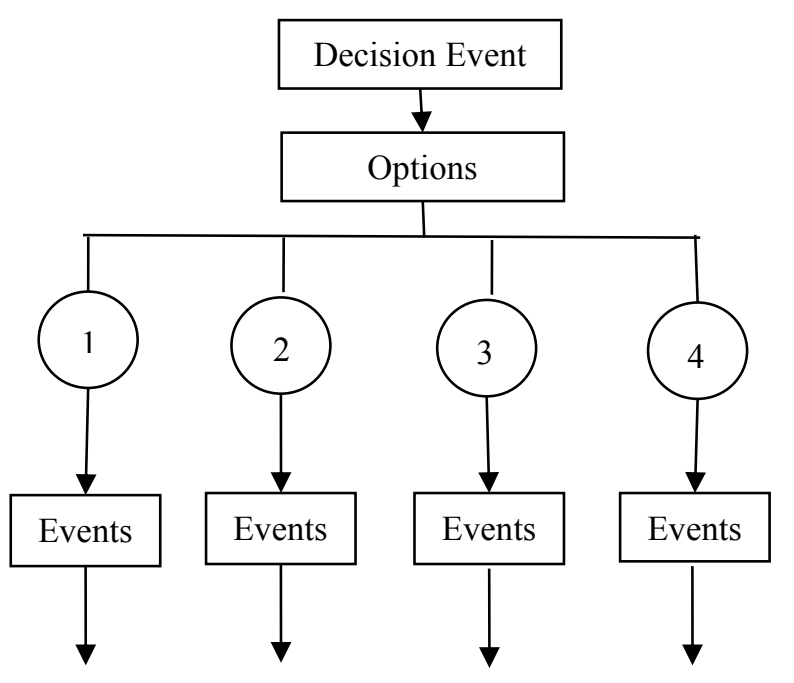

Fig. 3 Branching Model

The design of the branching mechanism is as follows: Each event in Pandora + has a branch level, option link and decision event serial attribute attached to it.

- The branch level is used to determine which level the event belongs to. An event can only belong to one branch level. The first branch level is always 0 (base Level). As a scenario branches, the branch level is incremented (see Fig. 3).

- The option link is used to determine the option to which the event belongs. The value of this attribute ranges from 1-4 only (see Fig. 3).

- The decision event serial is used to determine the parent decision event an event belongs to, which is essentially the decision point. The value is a unique value from the serial attribute belonging to a decision event (see Fig. 3).
When a scenario branches, events are retrieved from the database by using the branch level, option link and decision event serial to find events that match.

Event Loading Algorithm (Part of the Branching Algorithm above):

1) Get selected option from response by the trainee(s) (1-

\section{4) (option link)}

2) Get decision event for option

3) Get serial for decision event

4) Get current branch level

5) Increment branch level

6) Retrieve all events from the database with: serial in (3) matching the event decision event serial attribute, the branch level in (5) matching event branch level attribute and the option link in (1) matching the event option link attribute.

\section{EVALUATION}

A robust prototype version of the original Pandora system was trialled at the Emergency Planning College in York, UK in 2012 with a total of 13 Gold Commanders who were split into three groups, each group being trained for one day using the Pandora system. The experience of the Gold Commanders present, ranged from novice to experienced and the overall feedback was extremely positive. The fundamental approach taken by Pandora worked extremely well and trainees were clearly immersed in the environment throughout the entire training session. Details of that evaluation have been reported before in [14], [15]. Pandora $^{+}$has been used by a partner training company for a number of training events while under development, and is now available as a commercial product. In addition, the underpinning toolset will be developed further through forthcoming European research projects.

\section{CONCLUSIONS AND FUTURE WORK}

The Pandora+ system provides a leading-edge eLearning training environment which can be used to educate trainees in any topic where an event-based training scenario is relevant. The system is a toolset providing a rich multimedia training environment into which a scenario can be entered or uploaded and then executed, thus allowing it to be tailored to different domains. The system provides the trainer with a range of controls to manipulate the training before or during a training event. The focus of this is around the management of the events in an unfolding scenario in which the trainees have to make a sequence of strategic decisions, and a detailed explanation of the decision branching mechanism has been provided in this paper. This mechanism is not specific to crisis management and could be applied to similar problems requiring dynamic branching. 
Pandora $^{+}$has a variety of sophisticated controls to manipulate the stress of a trainee. Like all the facilities in Pandora $^{+}$the trainer has the option to use them as and when they offer value to the training event.. Enhanced training of this kind has the potential to deliver significant societal impacts. For example within crisis management alone, it could reduce casualty rates, enable faster and more efficient remediation, reduce loss of working time, reduce loss of productivity and improve coordination of expensive resources etc.

In terms of future work, at the moment the Pandora ${ }^{+}$ system requires that all the multimedia assets are downloaded to the client in advance of the training. The system also has to pause whilst the next set of events are loaded after a decision has been made. In future, in order to enhance efficiency and flexibility, a look ahead feature will be implemented so that prior to a decision event, the events, plus any associated media assets, will be downloaded for a fixed period of scenario time ahead, for all option choices, so that the event network can be updated more efficiently after a decision is made, ensuring that trainees remain immersed in the scenario.

The system is also being developed as a distributed, webenabled training environment, supporting a greater level of VR, to provide ongoing training and updates to Gold Commanders in the workplace, regular multi-agency coordination training events, and to support Recovery scenarios over longer-term periods.

Biometric inputs are being extended to include EEG inputs when measuring stress, and to investigate decision behaviours. These will be linked to the feedback and debrief mechanism, to help trainees understand and improve their decision-making processes, in particular in group and multiagency situations.

Also, as identified earlier, the toolset continues to be used and developed through ongoing European research projects, in which the authors are involved, and feedback from the commercial use of Pandora ${ }^{+}$will likewise be utilised in the continued development of the system.

\section{ACKNOWLEDGMENT}

The authors would like to thank colleagues from the other partners in the original PANDORA project for their contributions to the work presented, those partners being CEFRIEL (Italy), XLAB (Slovenia), FUB (Italy), UEL (U.K.), ORT (France) and EPC (U.K.). We would also wish to thank the EU for funding this original work under FP7-ICT-SEC2007-1 grant number 225387.

$\begin{array}{lll}\begin{array}{l}\text { Branch } \\ \text { Level 0 }\end{array} & \begin{array}{l}\text { Branch } \\ \text { Level 1 }\end{array} & \begin{array}{l}\text { Branch } \\ \text { Level 2 }\end{array} \\ \text { Decision } & \text { Events with } & \\ \text { Event } & \text { Decision Event } & \\ \text { (Serial: } & \text { Serial }=15 & \\ \text { UNIQUE } & \text { Branch level }=1 & \\ \text { e.g. 15 } & \text { Option Link }=1 & \end{array}$

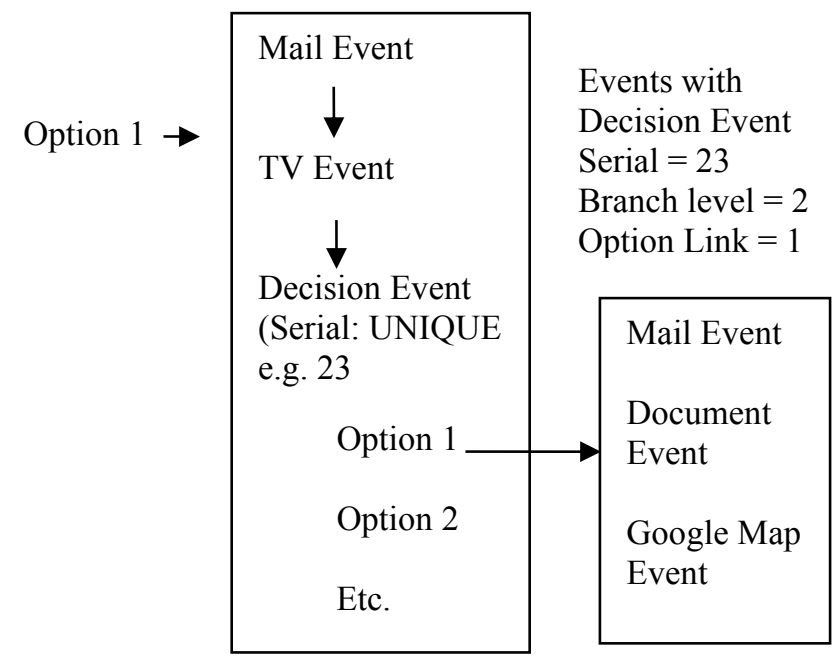

Events with

Option $2 \rightarrow \quad \begin{aligned} & \text { Decision Event } \\ & \text { Serial }=15 \\ & \text { Branch level }=1 \\ & \text { Option Link }=2\end{aligned}$

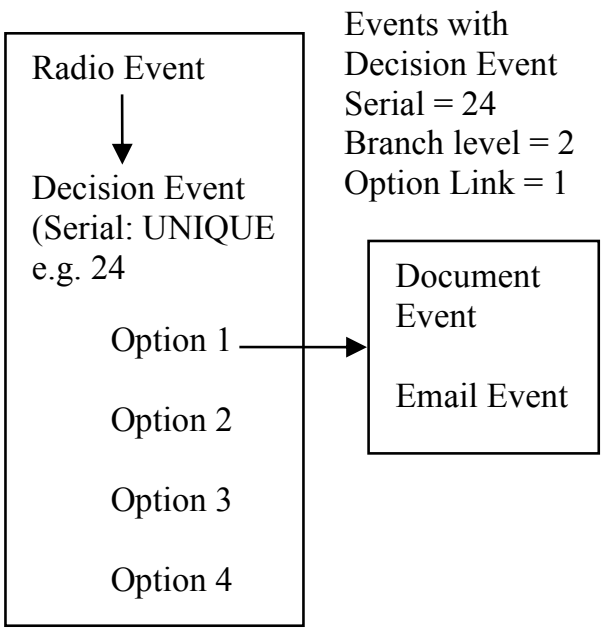

Fig. 4 Decision Event Branching Model 


\section{REFERENCES}

[1] Weaver, S., Dy, S.M., Rosen, M. A. "Team-training in healthcare: a narrative synthesis of the literature". BMJ Qual Saf 2014;23:359372 doi:10.1136/bmjqs-2013-001848.

[2] Taneja S., Pryor, M.G., Sewell, S., Recuero, A. M. "Strategic Crisis Management: A Basis for Renewal and Crisis Prevention”. Journal of Management Policy and Practice vol. 15(1) 2014.

[3] Clark Russell D., III. "Risk Taking in Groups: A Social Psychological Analysis", The Journal of Risk and Insurance. Vol. 41, No. 1, Mar., 1974, Page 75 of 75-92.

[4] Giora Keinan "Decision Making Under Stress: Scanning of Alternatives Under Controllable and Uncontrollable Threats" Journal of Personality and Social Psychology 1987, Vol. 52, No. 3,639-644.

[5] M. Crichton, R. Flin "Training for emergency management: tactical decision games. Journal of Hazardous Materials". 88 (2001) 255-266 Elsevier

[6] Victoria Guillén-Nieto and Marian Aleson-Carbonell. "Serious games and learning effectiveness: The case of It's a Deal!" Computers \& Education 58 (2012) 435-448 Elsevier.

[7] M. Graafland, J. M. Schraagen and M. P. Schijven. "Systematic review of serious games for medical education and surgical skills training" British Journal of Surgery 2012; 99: 1322-1330

[8] Ryan Flynn, Lachlan MacKinnon, Elizabeth Bacon, Janet Webb. Maritime City: "Using Games Technology to train Social Workers some initial results". Proceedings of ICEC 2011, 10th International Conference on Entertainment Computing, October 5 - October 8, 2011 Vancouver, Canada. Paper and poster presentation.

[9] Stephen R. Serge, Heather A. Priest, Paula J. Durlach, Cheryl I. Johnson. "The effects of static and adaptive performance feedback in game-based training" Computers in Human Behavior 29 (2013) 1150-1158.

[10] David R. Pratt and Matthias Spruill. "Games versus Virtual Simulation: An informal survey". SCS M\&S Magazine - 2011-01 (Jan).

[11] Hydra / Minerva User group 2010 http://www.hydraminerva.com/index.htm

[12] VectorCommand 2009 http://www.vectorcommand.com/

[13] Rankin, Amy, Joris Field, William Wong, Henrik Eriksson, and Chris Rooney. "Scenario Design for Training Systems in Crisis Management: Training Resilience Capabilities." In Scenario Design for Training Systems in Crisis Management: Training Resilience Capabilities. 2011.

[14] L. Bacon, L. MacKinnon, A. Cesta, G. Cortellessa. "Developing a Smart Environment for Crisis Management Training". Special edition of the Journal of Ambient Intelligence and Humanized Computing, entitled Smart Environments and Collective Computational Intelligence for Disaster Management. Vol 3, No 2. 2012. DOI: 10.1007/s12652-0120124-0.Available http://www.springerlink.com/content/r586h5354923/?MUD=MP

[15] Lachlan Mackinnon, Liz Bacon, Gabriella Cortellessa, Amedeo Cesta. "Using Emotional Intelligence in Training Crisis Managers: The Pandora Approach". The International Journal of Distance Education Technologies (IJDET) special issue on Emotional Intelligence for Online Learning, pages 66-95. Published by IGI Global. Issue 2, May 2013. http://www.igi-global.com/article/using-emotional-intelligencetraining-crisis/77841

[16] Cesta, A., Cortellessa, G., De Benedictis, R. and Strickland, K. AI*IA 2011.Using Planningfor Training Crisis Decision Makers. AI*IA-11. 12th International Conference of the Artificial Intelligence Italian Association. Palermo, Italy, September 15-17, 2011.

13 $^{\text {th }}$ LACCEI Annual International Conference: “Engineering Education Facing the Grand Challenges, What Are We Doing?" July 29-31, 2015, Santo Domingo, Dominican Republic 